\title{
Changes in Cropping Pattern of Selected Households of Wadi Programme in Korba District of Chhattisgarh, India
}

\author{
Parul Singh* , A.K. Gauraha and Kirti Sahu \\ Department of Agricultural Economics College of Agriculture, IGKV, Raipur, \\ Chhattisgarh, India \\ *Corresponding author
}

\section{A B S T R A C T}

\begin{tabular}{|l|}
\hline K e y w o r d s \\
$\begin{array}{l}\text { Cropping pattern, Wadi } \\
\text { programme, Cropping } \\
\text { intensity, Cropped area, } \\
\text { House holdes }\end{array}$ \\
\hline Article Info \\
\hline $\begin{array}{l}\text { Accepted: } \\
\text { 06 August } 2018 \\
\text { Available Online: } \\
\text { 10 September } 2018\end{array}$ \\
\hline
\end{tabular}

\begin{abstract}
The present study is based on impact assessment of wadi programme with the following objectives: To find out the changes in cropping pattern of selected household of wadi programme. One hundred twenty wadi farmers were selected randomly from Korba district for the year 2016-17, which were related to general characteristics of sample households and changes on cropping pattern of selected households before and after wadi programme. The major findings of this study revealed that The cropping intensity was observed to be 117.82 percent in before wadi and 154.62 percent in after wadi its increase about 36.81 percent out of total cropped area before wadi programme in kharif rabi and zaid crops occupied about 2.11 ha, 0.30 ha and 0.07 ha respectively and after wadi programme out of total cropped area kharif, rabi and zaid crops occupied about 2.18 ha, 0.64 ha and 0.50 ha respectively. In wadi programme farmers are including plantation crops like mango, lemon, and cashew and forest crops like babool and karonda.
\end{abstract}

\section{Introduction}

Notwithstanding the tradition of having trees on farmland and awareness among farmers about the benefits of tree-based farming systems, adoption of agro forestry in the country remained low for a long time. Information in the form of publications on traditional practices or newly developed systems did not result in large scale adoption of agro forestry. There were extension efforts and publications brought out to provide information on the physical arrangement of species that combined to form a system and their management. However, it was found that such information alone was inadequate to promote agro forestry as it requires several other considerations. This is evident in the success of an agro forestry based programme that has been implemented in several parts of the country. Known as the Wadi (orchard) programme, this comprehensive approach to rural development was designed and implemented by the BAIF Development Research Foundation. Besides the physical unit of the farm, the Wadi programme addresses a number of issues that have an indirect bearing on farm productivity. Wadi was first initiated via BAIF DHRUVA, by implementing orchard model for tribal development at tribal belt of Navsari and Valsad district of South Gujarat. In this model, 
the plantation of different fruit crops along with forest tree species around border line of orchard with holistic approach to obtain higher production. Increasing population has adversely affects the conservation of natural resources in most of the developing countries. In India most of the rural people uses wood for their fuel and fodder purpose resulting declining forest resources. It not only threatens the existence of our precious flora and fauna but also affect a large number of tribal communities who inhabitants over 10 per cent of the Indian population and are considered as traditional custodians of forest resource. Over 2025 million families in live of 250 different tribal communities have been living on the edge of the natural forests and depending their livelihood through collection of a wide range of fruits, fibre, honey, lac, gum and medicinal herbs. With increasing in population growth there has been tremendous pressure on the supply of forest products, indiscriminate felling of trees causing denudation of forest resources and severely affected the livelihood of the tribal. The adivasi population in India constitutes about 8.14 per cent of the total population or approximately 85 million people (2001 Census). These households traditionally derive sustenance through forestry, hunting and primitive agriculture practices (Phansalkar and Verma, 2005). However, fast depleting forest, and natural resources, land erosion, lack of access to basic health and hygiene have made seasonal migration into nearby cities a virtual necessity. In the cities these landless workers have to live in deplorable conditions and often get exploited by middlemen. They are also not able to claim benefits being offered by the state governments due to a lack of identity (Hooza, 2004). Ineffective labour laws make their situation (especially women) very difficult. Large sections (approx 36\%) of the tribals continue to be in a state of deprivation (Pandya, 1988). Over the last two decades, the Wadi has been transformed into a multidisciplinary programme for rehabilitation. The essence is to provide sustainable livelihood from an acre (0.4 ha) of land for the participating poor family. Each Wadi owner can earn Rs. 20,000-25,000 annually from this small plot of land, once the trees start bearing fruits. There are further opportunities to enhance the income through other supplementary activities.

\section{Materials and Methods}

\section{Sampling procedure}

The detailed sampling procedure of selection of the district, block, and villages Wadi development programme are presented under following sub-section.

\section{Selection of district}

Out of 27 Districts of Chhattisgarh state, Korba district has been selected purposively for the study.

\section{Selection of study area}

Out of 27 districts of Chhattisgarh, Korba district was selected purposively for the study. Korba district having 5 blocks Korba, Kartala, Katghora, Pali and Pondiuproda. Out of 5 blocks of Korba district, Pali block was selected purposively for the study.

\section{Selection of villages}

In Pali block, Wadi programme was undertaken in 23 villages out of these five villages namely Ganeshpur, Kapot, Damiya, Kartali, and Banbandha was purposively selected for the study.

\section{Selection of households}

In five selected villages the number of beneficiaries was 240 out of these fifty percent 
beneficiaries of Wadi programme was selected randomly, Thus the total number of selected households was 120 in number, Sample households was selected to adopt probability proportionate to size sampling techniques.

\section{Method of enquiry and Data collection:}

The study required primary data as well as secondary data. The primary data from the farmers was collected through personal interview method Interview-schedule,

Semi- participant Observation, Case study, Focused Group Discussion, Photography and Video recording with the help of wellprepared pretested schedule and questionnaire for the year 2010-11 to 2015-16.

The secondary data was collected through different Government offices such as Department of Agriculture, Government of Chhattisgarh, Documents of Raigarh Sahyog Samiti, Wadi and NABARD; Journals eresources, Magazines, Census data and scan through various sources.

\section{Period of Enquiry}

The enquiry conducted during the year 20162017 in Pali bock of Korba district.

\section{Analytical Procedure}

To analyse the primary data simple averages and percentages method will be used looking to the quantity quality of total data.

\section{Cropping Intensity}

Ratio of Gross cropped area and total cropped area.

$$
\begin{gathered}
\text { Gross Cropped Area } \\
\text { Cropping Intensity }=\text {------------- X } 100 \\
\text { Net Cropped Area }
\end{gathered}
$$

\section{Results and Discussion}

Changes on cropping pattern, income and employment pattern of selected households

\section{Cropping pattern}

The Table 1 cropping pattern before Wadi programme reveals that in kharif, paddy on an average occupied the largest area (72.83 per cent) followed by maize (16.35 per cent) redgram (10.82 per cent).

In rabi season lathyrus on an average occupies maximum area (33.17 per cent), followed by gram (29.62 per cent), and urd (20.26 per cent). Okra on an average occupied the largest area ( 57.14 per cent) followed by bottle gourd (42.86 per cent) in zaid.

The average cropping intensity in the study area was observed 117.82 per cent. Cropping intensity shows a decreasing trend with the rise in farms. The cropping intensity was follow to be $122.95,122.50,114.64$ and 112.72 per cent at marginal, small, medium and large farms, respectively.

The maximum cropped area was found to be during kharif among all the size holdings. It may be observed from analysis that most of the respondents in the study area were interested to grow paddy during kharif season.

Cropping pattern in the project area revealed the dominance of traditional crops like paddy, Wheat, Maize and pulses (Redgram, Urd, Moong, Gram) and to some extent oil seeds (mustard) and vegetable, but with emergence of Wadi component the area under horticulture crops has acquired a significant share of $20 \%$ of the total area under cultivation under these crops Mango/Cashew nut/Lemon yield per acre measures the crop productivity which shows positive change mostly in the owned land + wadi land than forest/revenue land. 
Table.1 Cropping pattern of sample households (before Wadi)

\begin{tabular}{|c|c|c|c|c|c|c|}
\hline \multirow{3}{*}{$\frac{\text { S. No. }}{\text { A }}$} & \multirow{3}{*}{\begin{tabular}{|l|} 
Particular \\
Kharif
\end{tabular}} & \multirow{2}{*}{\multicolumn{4}{|c|}{ Farm size }} & ha/farm) \\
\hline & & & & & & \multirow[t]{2}{*}{ overall } \\
\hline & & Marginal & Small & Medium & Large & \\
\hline \multirow[t]{2}{*}{$\mathbf{A}$} & Paddy & 0.48 & 1.45 & 2.56 & 4.30 & 1.53 \\
\hline & & $(78.69)$ & $(72.50)$ & $(64.65)$ & (78.18) & $(72.83)$ \\
\hline \multirow[t]{2}{*}{ B } & Redgram & 0.07 & 0.05 & 0.80 & 0.70 & 0.23 \\
\hline & & (11.48) & $(2.50)$ & $(20.20)$ & (12.73) & $(10.82)$ \\
\hline \multirow[t]{5}{*}{ C } & Maize & 0.06 & 0.50 & 0.60 & 0.50 & 0.34 \\
\hline & & $(9.84)$ & $(25.00)$ & $(15.15)$ & $(9.09)$ & $(16.35)$ \\
\hline & sub total & 0.61 & 2.00 & 3.96 & 5.50 & 2.11 \\
\hline & & $(81.33)$ & $(81.63)$ & $(87.22)$ & $(88.70)$ & (85.08) \\
\hline & & (100.00) & (100.00) & (100.00) & (100.00) & $(100.00)$ \\
\hline B & Rabi & & & & & \\
\hline \multirow[t]{2}{*}{ A } & Wheat & 0.00 & 0.00 & 0.00 & 0.06 & 0.01 \\
\hline & & $(0.00)$ & $(0.00)$ & $(0.00)$ & $(16.67)$ & $(2.15)$ \\
\hline \multirow[t]{2}{*}{ B } & b. Mustard & 0.00 & 0.00 & 0.05 & 0.05 & 0.02 \\
\hline & & $(0.00)$ & $(0.00)$ & (16.13) & (13.89) & $(6.52)$ \\
\hline \multirow[t]{10}{*}{ C } & c. Lathyrus & 0.06 & 0.20 & 0.15 & 0.05 & 0.10 \\
\hline & & $(42.86)$ & $(44.44)$ & $(48.39)$ & (13.89) & $(33.17)$ \\
\hline & d. Urd & 0.06 & 0.09 & 0.01 & 0.03 & 0.06 \\
\hline & & $(42.86)$ & $(20.00)$ & (3.23) & $(8.33)$ & $(20.26)$ \\
\hline & e. Moong & 0.00 & 0.00 & 0.05 & 0.17 & 0.03 \\
\hline & & $(0.00)$ & $(0.00)$ & $(16.13)$ & $(47.22)$ & $(8.29)$ \\
\hline & f. Gram & 0.02 & 0.16 & 0.05 & 0.00 & 0.09 \\
\hline & & $(14.29)$ & $(35.56)$ & $(55.56)$ & $(0.00)$ & $(29.62)$ \\
\hline & Sub Total & 0.14 & 0.45 & 0.31 & 0.36 & 0.30 \\
\hline & & (18.66) & $(18.36)$ & $(6.82)$ & $(5.80)$ & (12.09) \\
\hline \multirow[t]{7}{*}{ C } & ZAID & & & & & \\
\hline & a. Bottlegaurd & 0.00 & 0.00 & 0.09 & 0.10 & 0.03 \\
\hline & & $(0.00)$ & $(0.00)$ & (33.33) & (29.41) & $(42.86)$ \\
\hline & b. Okra & 0.00 & 0.00 & 0.18 & 0.05 & 0.04 \\
\hline & & $(0.00)$ & $(0.00)$ & (66.67) & $(14.71)$ & $(57.14)$ \\
\hline & sub total & 0.00 & 0.00 & 0.27 & 0.34 & 0.07 \\
\hline & & $(0.00)$ & $(0.00)$ & $(5.94)$ & $(5.48)$ & $(2.82)$ \\
\hline \multirow[t]{2}{*}{ D } & $\begin{array}{l}\text { Total cropped area } \\
(A+B+C)\end{array}$ & 0.75 & 2.45 & 4.54 & 6.20 & 2.48 \\
\hline & & $(100.00)$ & $(100.00)$ & (100.00) & (100.00) & $(100.00)$ \\
\hline $\mathbf{E}$ & Net cultivated area (A) & 0.61 & 2.00 & 3.96 & 5.50 & 2.11 \\
\hline $\mathbf{F}$ & $\begin{array}{l}\text { Double cropped area } \\
(\mathrm{B}+\mathrm{C})\end{array}$ & 0.14 & 0.45 & 0.58 & 0.70 & 0.38 \\
\hline $\bar{G}$ & Cropping intencity (\%) & 122.95 & 122.50 & 114.64 & 112.72 & 117.82 \\
\hline
\end{tabular}

Note: Figures in the parenthesis indicate the percentages to the total. 
Table.2 Cropping pattern of sample households (After Wadi)

(in ha)

\begin{tabular}{|c|c|c|c|c|c|c|}
\hline S. No & Particulars & \multicolumn{4}{|c|}{ Farm Size } & \multirow[t]{2}{*}{ Overall } \\
\hline $\mathbf{A}$ & KHARIF & Marginal & SMALL & MEDIUM & LARGE & \\
\hline \multirow[t]{2}{*}{ a. } & Paddy & 0.50 & 1.50 & 2.80 & 4.50 & 1.62 \\
\hline & & (71.43) & (73.53) & $(67.96)$ & $(80.53)$ & (74.03) \\
\hline \multirow[t]{2}{*}{ b. } & Maize & 0.00 & 0.04 & 0.40 & 0.20 & 0.09 \\
\hline & & $(0.00)$ & $(1.96)$ & $(9.71)$ & (3.58) & $(4.13)$ \\
\hline \multirow[t]{3}{*}{ c. } & Redgram & 0.00 & 0.08 & 0.30 & 0.13 & 0.10 \\
\hline & & $(0.00)$ & (3.92) & (7.28) & (2.33) & $(4.47)$ \\
\hline & PLANTATION CROPS & & & & & \\
\hline \multirow[t]{2}{*}{$\underline{\mathbf{A}}$} & Mango & 0.15 & 0.10 & 0.15 & 0.20 & 0.14 \\
\hline & & (21.43) & $(4.90)$ & (3.64) & (3.58) & $(6.26)$ \\
\hline \multirow[t]{2}{*}{ b. } & Cashew & 0.01 & 0.05 & 0.05 & 0.10 & 0.04 \\
\hline & & (1.43) & (2.45) & $(1.21)$ & (1.79) & $(1.84)$ \\
\hline \multirow[t]{3}{*}{ c. } & Lemon & 0.02 & 0.05 & 0.05 & 0.10 & 0.04 \\
\hline & & (2.86) & (2.45) & (1.21) & (1.79) & $(2.01)$ \\
\hline & INTERCROP VEGETABLE & & & & & \\
\hline \multirow[t]{2}{*}{$\mathbf{A}$} & Brinjal & 0.01 & 0.03 & 0.12 & 0.20 & 0.05 \\
\hline & & (1.43) & (1.47) & (2.91) & (3.58) & $(2.24)$ \\
\hline \multirow[t]{2}{*}{ b. } & Chilli & 0.00 & 0.03 & 0.12 & 0.10 & 0.04 \\
\hline & & $(0.00)$ & (1.47) & (2.91) & (1.79) & $(1.76)$ \\
\hline \multirow[t]{3}{*}{ c. } & Tomato & 0.01 & 0.08 & 0.01 & 0.01 & 0.03 \\
\hline & & $(0.71)$ & (3.92) & $(0.24)$ & $(0.18)$ & $(1.49)$ \\
\hline & FORESTRY CROP & & & & & \\
\hline \multirow[t]{2}{*}{$\mathbf{A}$} & Karonda & 0.00 & 0.03 & 0.01 & 0.04 & 0.02 \\
\hline & & $(0.00)$ & (1.47) & $(0.24)$ & $(0.72)$ & $(0.79)$ \\
\hline \multirow[t]{5}{*}{ b. } & Babool & 0.00 & 0.05 & 0.01 & 0.01 & 0.02 \\
\hline & & $(0.00)$ & (2.45) & $(0.24)$ & $(0.14)$ & $(0.98)$ \\
\hline & Sub total & 0.70 & 2.04 & 4.12 & 5.59 & 2.18 \\
\hline & & $(44.30)$ & $(61.26)$ & (75.59) & (79.62) & $(65.26)$ \\
\hline & & $(100.00)$ & $(100.00)$ & $(100.00)$ & $(100.00)$ & $(100.00)$ \\
\hline B & RABI & & & & & \\
\hline \multirow[t]{2}{*}{$\underline{\mathbf{A}}$} & Lathyrus & 0.08 & 0.06 & 0.10 & 0.03 & 0.07 \\
\hline & & (16.84) & (8.70) & (10.20) & (3.57) & (10.88) \\
\hline \multirow[t]{2}{*}{ B } & Wheat & 0.06 & 0.08 & 0.20 & 0.07 & 0.09 \\
\hline & & (12.47) & (11.59) & (20.41) & (8.33) & $(13.54)$ \\
\hline \multirow[t]{2}{*}{$\mathbf{C}$} & Moong & 0.01 & 0.07 & 0.10 & 0.06 & 0.05 \\
\hline & & (2.08) & (10.14) & $(10.20)$ & (7.14) & (7.75) \\
\hline \multirow[t]{3}{*}{ D } & Urd & 0.03 & 0.04 & 0.00 & 0.10 & 0.04 \\
\hline & & $(6.24)$ & $(5.80)$ & $(0.00)$ & $(11.90)$ & $(5.79)$ \\
\hline & PLANTATION CROPS & & & & & \\
\hline A & Mango & 0.15 & 0.10 & 0.15 & 0.20 & 0.14 \\
\hline
\end{tabular}




\begin{tabular}{|c|c|c|c|c|c|c|}
\hline & & (31.19) & (14.49) & $(15.31)$ & $(23.81)$ & $(21.21)$ \\
\hline \multirow[t]{2}{*}{ B } & Cashew & 0.01 & 0.05 & 0.05 & 0.10 & 0.04 \\
\hline & & $(2.08)$ & (7.25) & $(5.10)$ & (11.90) & $(6.22)$ \\
\hline \multirow[t]{3}{*}{$\mathbf{C}$} & Lemon & 0.02 & 0.05 & 0.05 & 0.10 & 0.04 \\
\hline & & $(4.16)$ & (7.25) & $(5.10)$ & $(11.90)$ & $(6.81)$ \\
\hline & INTERCROP VEGETABLE & & & & & \\
\hline \multirow[t]{2}{*}{ A } & Radish & 0.04 & 0.10 & 0.10 & 0.04 & 0.07 \\
\hline & & $(8.32)$ & (14.49) & $(10.20)$ & $(4.76)$ & $(10.94)$ \\
\hline \multirow[t]{2}{*}{ B } & Tomato & 0.02 & 0.04 & 0.20 & 0.10 & 0.06 \\
\hline & & $(4.16)$ & $(5.80)$ & (20.41) & (11.90) & $(9.34)$ \\
\hline \multirow[t]{3}{*}{ C } & Colecrops & 0.06 & 0.10 & 0.03 & 0.04 & 0.05 \\
\hline & & (12.47) & $(0.00)$ & $(3.06)$ & $(4.76)$ & $(0.02)$ \\
\hline & FORESTRY CROP & & & & & \\
\hline \multirow[t]{2}{*}{ A } & Karonda & 0.00 & 0.03 & 0.01 & 0.04 & 0.02 \\
\hline & & $(0.00)$ & $(4.35)$ & $(1.02)$ & $(4.76)$ & $(2.68)$ \\
\hline \multirow[t]{4}{*}{$\mathbf{B}$} & Babool & 0.00 & 0.05 & 0.01 & 0.01 & 0.02 \\
\hline & & $(0.00)$ & $(7.25)$ & $(1.02)$ & $(0.95)$ & $(3.31)$ \\
\hline & Sub total & 0.48 & 0.69 & 0.98 & 0.84 & 0.64 \\
\hline & & $(30.37)$ & (20.72) & (17.98) & (11.96) & (19.16) \\
\hline \multirow[t]{2}{*}{$\mathrm{C}$} & ZAYAD & & & & & \\
\hline & PLANTATION CROPS & & & & & \\
\hline \multirow[t]{2}{*}{ A } & Mango & 0.15 & 0.10 & 0.15 & 0.20 & 0.14 \\
\hline & & $(36.41)$ & $(16.13)$ & $(41.67)$ & $(34.01)$ & $(27.22)$ \\
\hline \multirow[t]{2}{*}{ B } & Cashew & 0.01 & 0.05 & 0.05 & 0.10 & 0.04 \\
\hline & & $(2.43)$ & $(8.06)$ & (13.89) & $(17.01)$ & (7.98) \\
\hline \multirow[t]{3}{*}{$\mathbf{C}$} & Lemon & 0.01 & 0.05 & 0.05 & 0.10 & 0.04 \\
\hline & & (2.43) & (8.06) & $(13.89)$ & $(17.01)$ & $(7.98)$ \\
\hline & FORESTRY CROP & & & & & \\
\hline \multirow[t]{2}{*}{ A } & Karonda & 0.00 & 0.03 & 0.01 & 0.04 & 0.02 \\
\hline & & $(0.00)$ & $(4.84)$ & $(2.78)$ & $(6.80)$ & $(3.45)$ \\
\hline \multirow[t]{3}{*}{$\mathbf{B}$} & Babool & 0.00 & 0.05 & 0.01 & 0.01 & 0.02 \\
\hline & & $(0.00)$ & $(8.06)$ & $(2.78)$ & $(1.36)$ & $(4.25)$ \\
\hline & INTERCROP VEGETABLE & & & & & \\
\hline \multirow[t]{2}{*}{ A } & Cucumber & 0.04 & 0.04 & 0.05 & 0.07 & 0.04 \\
\hline & & $(9.71)$ & $(6.45)$ & (13.89) & $(11.90)$ & $(8.88)$ \\
\hline \multirow[t]{4}{*}{ B } & Bottele Guard & 0.20 & 0.30 & 0.04 & 0.07 & 0.20 \\
\hline & & $(48.54)$ & (48.39) & (11.11) & $(11.90)$ & $(40.24)$ \\
\hline & Sub Total & 0.41 & 0.62 & 0.36 & 0.59 & 0.50 \\
\hline & & (25.94) & (18.61) & $(6.60)$ & $(8.40)$ & (14.97) \\
\hline $\mathbf{D}$ & TOTAL CROPPED AREA $(\mathrm{A}+\mathrm{B}+\mathrm{C})$ & 1.58 & 3.33 & 5.45 & 7.02 & 3.34 \\
\hline $\mathbf{E}$ & NET CULTIVATED AREA(A) & 0.68 & 2.04 & 4.12 & 5.59 & 2.18 \\
\hline $\mathbf{F}$ & DOUBLE CROPPED AREA(B+C) & 0.90 & 1.29 & 1.33 & 1.44 & 1.16 \\
\hline$\overline{\mathbf{G}}$ & CROPPING INTENSITY & 232.06 & 163.24 & 133.08 & 125.65 & 154.62 \\
\hline
\end{tabular}

Note: Figures in the parenthesis indicate the percentages to the total 
Table.3 Percent Change in Cropping Pattern over Year in ha

\begin{tabular}{|l|c|c|c|}
\hline Particulars & Before & After & \% Change \\
\hline Kharif & 1.48 & 1.77 & 19.6 \\
\hline Rabi & 0.16 & 0.4 & 150 \\
\hline Zaid & 0.08 & 0.32 & 300 \\
\hline Total cropped area & 1.72 & 2.7 & 56.98 \\
\hline Net Cultivated area & 1.48 & 1.72 & 16.21 \\
\hline Double Cropped area & 0.24 & 0.72 & 200 \\
\hline Cropping intensity & 116.22 & 156.98 & 35.07 \\
\hline
\end{tabular}

This shows the role of incentive on their own land/wadi the details are shown in the Table 2 reveals that in kharif, paddy on an average occupied the largest area (71.43 per cent) followed by plantation crop (10.11 per cent) inter crop vegetable (5.49 per cent). In rabi season plantation crop on an average occupies maximum area (34.24 per cent), followed by wheat (13.85 per cent), inter crop vegetable (20.03 per cent).Inter crop vegetable bottle guard on an average occupied the largest area (49.12 per cent) followed by Plantation crop (43.18 per cent) in zaid. The average cropping intensity in the study area was observed 154.62 per cent. Cropping intensity shows increasing trend with the rise in wadi. The cropping intensity was follow to be 232.06 , 163.24, 133.08 and 125.65 per cent at marginal, small, medium and large farms, respectively. The maximum cropped area was found to be during kharif among all the size holdings.

Percent change in cropping pattern over year is presented in Table 3. The total Percent change in cropping pattern was highest in zaid 300 per cent followed by rabi 150 per cent and 19.6 per cent in kharif. Percent change in total cropped area, net cultivated area, double cropped area, cropping intensity, are 56.98 per cent, 16.21 per cent, 200 per cent and 35.07 per cent respectvelly.

Out of total cropped area before wadi programme in kharif, rabi and zaid crops occupied about 2.11 ha., 0.30 ha and 0.07 ha., respectively. After wadi programme out of total cropped area kharif, rabi and zaid crops occupied about 2.18 ha., 0.64 ha and 0.50 ha., respectively. Total cropped area before wadi programme in kharif was covered under in kharif, paddy on an average occupied the largest area (72.83 per cent) followed by maize (16.35 per cent). In Rabi season lathyrus on an average occupies maximum area (33.17 per cent), followed by gram (29.62 per cent). Okra on an average occupied the largest area (57.14 per cent) followed by bottle gourd (42.86 per cent) in zaid. Total cropped area after wadi programme in kharif was covered under in kharif, paddy on an average occupied the largest area (74.03 per cent) followed by plantation crop (10.11 per cent) inter crop vegetable (5.49 per cent). In rabi season plantation crop on an average occupies maximum area (34.24 per cent), followed by wheat (13.85 per cent), inter crop vegetable (20.03 per cent).Inter crop vegetable bottle guard on an average occupied the largest area (49.12 per cent) followed by Plantation crop (43.18 per cent) in zaid. The average cropping intensity observed in the study area was 117.82 per cent before wadi programme and 154.62 per cent after the wadi programme. The total percent change in cropping pattern was highest in zaid 300 per cent followed by Rabi 150 per cent and 19.6 per cent in kharif. Percent change in total cropped area, net cultivated area, double cropped area, cropping intensity, are 56.98 
per cent, 16.21 per cent, 200 per cent and 35.07 per cent respectively.

\section{References}

BAIF (1982) Rehabilitation Experiences Wadi Programme District Valsad, Navsari and Dangs

BAIF Development Research Foundation (1998) Integrated Rural Development for Sustainable Development BAIF 1998. PP. 144-105.
BAIF Development Research Foundation Annual Reports - 1999-2000, 20012002-2003.

BAIF Development Research Foundation Progress Reports of Comprehensive Tribal Development.

Bhatt Anil (1987) Rehabilitation Approach to Poverty, Alleviation of Tribals, Indian Institute of Management (IIM), 1987.

D.D.O. (Dangs) Dangs Development Report

Tandel B.M, Y.N. Tandel, R.M. Naik and C.K. Timbadiya (2014) Wadi: A boon for sustainable livelihood, Agric. Update, 9(2): 204-206.

\section{How to cite this article:}

Parul Singh, A.K. Gauraha and Kirti Sahu. 2018. Changes in Cropping Pattern of Selected Households of Wadi Programme in Korba District of Chhattisgarh, India. Int.J.Curr.Microbiol.App.Sci. 7(09): 529-536. doi: https://doi.org/10.20546/ijcmas.2018.709.063 